\title{
Piezoelectrically Driven and Sensed Micromirrors with Extremely Large Scan Angles and Precise Closed-Loop Control ${ }^{+}$
}

\author{
Shanshan Gu-Stoppel *, Thorsten Giese, Hans-Joachim Quenzer, Ulrich Hofmann \\ and Wolfgang Benecke \\ MEMS Application, Fraunhofer Institute for Silicon Technology, Itzehoe 25524, Germany; \\ thorsten.giese@isit.fraunhofer.de (T.G.); hans-joachim.quenzer@isit.fraunhofer.de (H.-J.Q.); \\ ulrich.hofmann@isit.fraunhofer.de (U.H.); wolfgang.benecke@isit.fraunhofer.de (W.B.) \\ * Correspondence: shanshan.gu-stoppel@isit.fraunhofer.de; Tel.: +49-4821-171-424 \\ + Presented at the Eurosensors 2017 Conference, Paris, France, 3-6 September 2017.
}

Published: 16 August 2017

\begin{abstract}
This paper reports piezoelectrically driven and sensed micromirrors achieving extremely large total optical scan angle of $106^{\circ}$ and high frequency of $45 \mathrm{kHz}$ at $22 \mathrm{~V}$. To reach such large angles, extensive FEM-simulations relating to fracture strength of poly-Si, of which these micromirrors primarily consist, have been performed. Meanwhile, high Q-factor of more than 5000 under ambient conditions and low power consumption of $10.3 \mathrm{~mW}$ were measured. Moreover the piezoelectric elements enable position sensing and based on the feedback signals closed-loop control has been developed.
\end{abstract}

Keywords: micromirror; piezoelectric; closed-loop control; fracture behavior

\section{Introduction}

Piezoelectric micromirrors combine advantages of high torque and low power consumption. Mostly piezoelectric micromirrors are driven by beam actuators, which consume power due to their bending motions. Considering a micromirror plate and actuators as an entire system the power should be consumed primarily for mirror torsion than actuator bending to raise the efficiency, which can be enabled by mechanical amplification using a leverage mechanism [1]. However, previous works showed the maximum achievable scan angles of piezoelectric micromirrors are strongly affected by the maximum breaking strength of the used material [2]. Thus, fracture strength of poly-Si, which is the basic material for micromirrors in this work, has been studied by FEM-simulations for optimizing the mirror designs. The achieved large scan angles of the mirrors have proven the results of the design optimization. A closed-loop control for piezoelectric micromirrors based on capacitive sensors was reported in [3]. Also integrated piezoresistive sensors were described in [4]. Despite the great sensor sensitivities hybrid integration of capacitive sensors and expensive fabrication of piezoresistive sensors remain their tradeoffs. Thus, integrated piezoelectric sensors, which cost no extra fabrication step, and appropriate closed-loop control have been studied and will be shown in this work.

\section{Design, FEM Simulation and Fabrication}

\subsection{Basic Design}

The presented micromirrors possess two piezoelectric beams (actuator and sensor) surrounding a central circular mirror plate, which are linked to each other by the connecting bars and torsion bars 
(Figure 1a). The piezoelectric material, PZT, is sandwiched between an upper and bottom electrode of $\mathrm{Au}$ and Pt. The mirror plate, beams and bars mainly consist of poly-Si, while $\mathrm{SiO}_{2}$ and thin $\mathrm{Al}$ layers serve as isolation layers and mirror surface, respectively (Figure 1b).

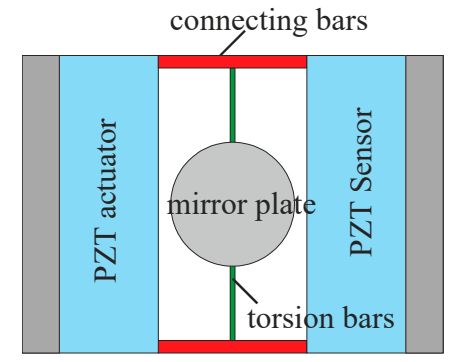

(a)

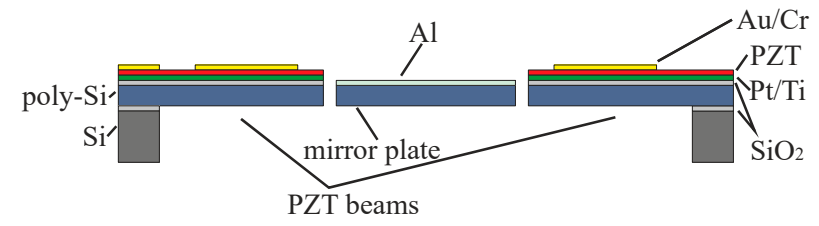

(b)

Figure 1. Sketch of the basic design S of 1D micromirror: (a) Top view; (b) Cross section.

The PZT beams not only drive the mirror but also amplify its displacement as levers. This mechanical efficiency of the system has been verified by the Laser-Doppler-Vibrometry measurements and the result will be shown in Section 3.

\subsection{Fracture Behavior and Design Optimization}

As above mentioned, previous work already showed that due to the large enough torque delivered by PZT actuators, the achievable scan angles are usually limited by the fracture strength of poly-Si, of which the micromirrors primarily consist. FEM-simulations have been thus performed to localize the cracking origins of the micromirrors and quantify the mechanical stress causing such device failures. Figure 2 shows the maximum Von Mises Stress of about $1.8 \mathrm{GPa}$ appears on the torsion bar and adjacent to the connecting bar, if a scan angle of $15^{\circ}$ the micromirror with design $\mathrm{S}$ is achieved.

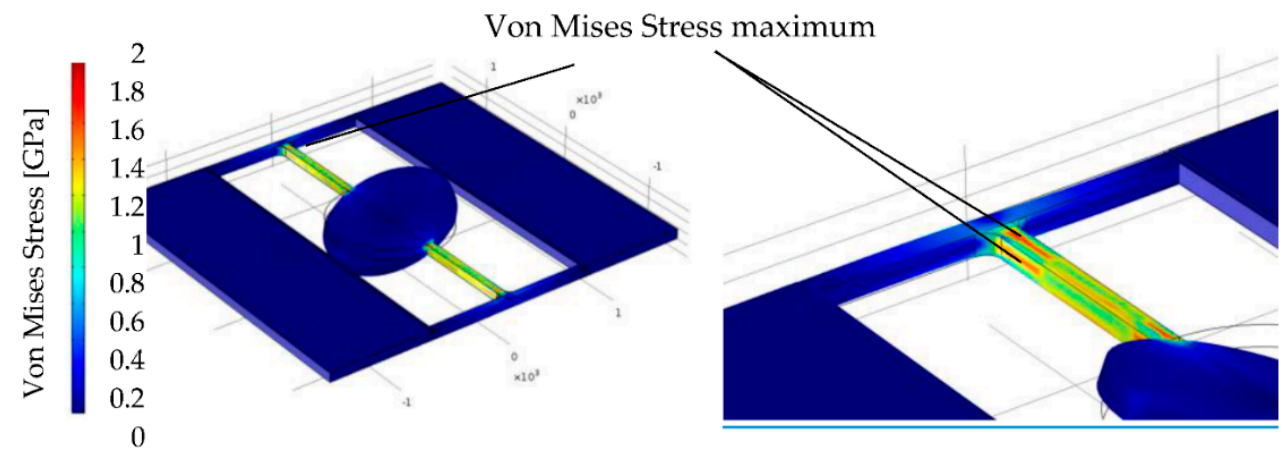

Figure 2. Von Mises Stresses of 1.8 GPa in design S, when these mirrors achieve a same mechanical tilting angle of $15^{\circ}$.

According to work [5] the location of stress maximum has been identified as the crack origin and design optimization has been performed by tuning the springs geometries, which allow the micromirrors to reach the same scan angles, while the maximum Von Mises Stresses are reduced, as Figure 3 shows. Design E5 und E4 apply different rounding, meandering springs as torsion bars and the maximum Von Mises Stresses are reduced to 1.6 GPa and 1.4 GPa. 


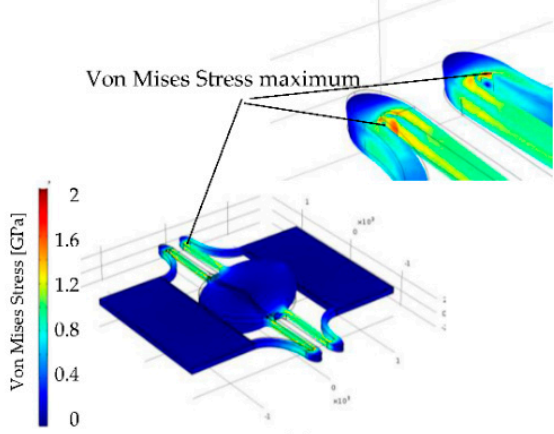

(a)

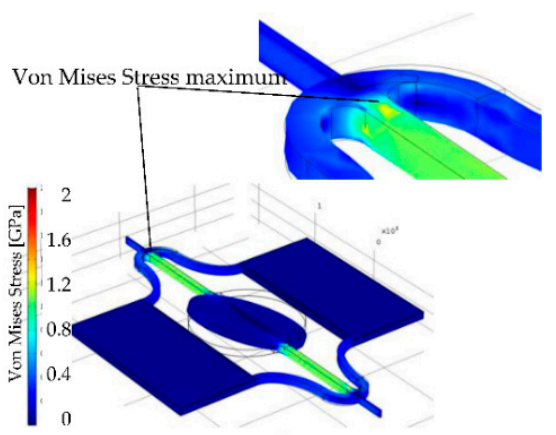

(b)

Figure 3. Design optimization: Different maximum Von Mises Stresses in different mirrors, when these mirrors achieve a same mechanical tilting angle of 15: (a) Von Mises stress maximum of 1.6 GPa in design E5; (b) Von Mises stress maximum of 1.4 GPa in design E4.

\section{Measurement Results}

The leverage amplification effect was proven by LDV and the recorded results are shown in Figure 4. The mirror plate has much larger displacement than the actuators, when they are driven in the torsional mode and the amplification factor approximates 30 .

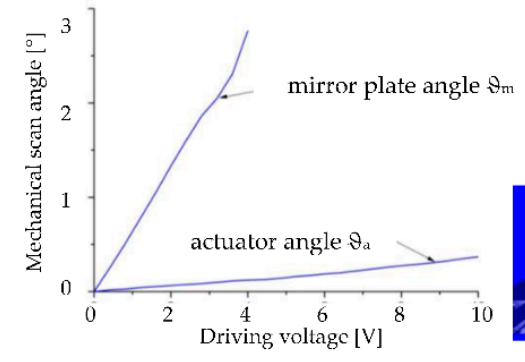

(a)

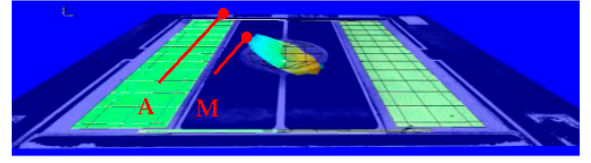

(b)

Figure 4. LDV measurement results of micromirror with design $\mathrm{S}$ (measure points are $\mathrm{A}$ and $\mathrm{M}$ ): (a) Mechanical scan angles of the mirror plate and the actuators; (b) Measurement recording of a deflected micromirror with scan angle of $2.5^{\circ}$ measured at point $\mathrm{M}$ and the actuators with scan angle of about $0.08^{\circ}$ measured at point $\mathrm{A}$.

The characterization results of different three design types in Figures 2 and 3 are shown in the following table (Table 1).

Table 1. The characterization results of Design S, E4 and E5.

\begin{tabular}{cccccccc}
\hline Design & $\begin{array}{c}\text { Aperture } \\
\text { Diameter } \\
{[\mathbf{m m}]}\end{array}$ & $\begin{array}{c}\text { Driving } \\
\text { Voltage } \\
{[\mathbf{V}]}\end{array}$ & $\begin{array}{c}\text { Full Optical } \\
\text { Scan Angle [] }\end{array}$ & $\begin{array}{c}\text { Simulated } \\
\text { Frequency } \\
{[\mathbf{k H z}]}\end{array}$ & $\begin{array}{c}\text { Measured } \\
\text { Frequency } \\
{[\mathbf{k H z}]}\end{array}$ & $\begin{array}{c}\text { Power } \\
\text { Consumption } \\
{[\mathbf{m W}]}\end{array}$ & Q-Factor \\
\hline S & 1 & 22 & 106.3 & 47.1 & 45.1 & 10.3 & 5149 \\
E4 & 1.2 & 25 & 86.7 & 28.1 & 26.8 & 13.5 & - \\
E5 & 1.2 & 20 & 104.2 & 34.1 & 31.2 & 5.8 & - \\
\hline
\end{tabular}

The last point of this work is to investigate position sensing and closed-loop control of the micromirror. One of the position sensing approaches is to use one of the PZT cantilever as the position sensor, while the second one severs as the actuator. Figure 5a shows the PZT sensing signal in frequency domain. After a simple signal processing of 64 averaging this signal shows already a good signal quality of $45 \mathrm{~dB}$ SNR, while the mechanical scan angle was only $0.7^{\circ}$. Since this micromirror can achieve a mechanical scan angle of $26.5^{\circ}$ meaning a full field of view of $106^{\circ}$, the total sensing resolution is larger than 12 bit [5]. Based on this great signal quality closed-loop control has been developed, as Figure $5 \mathrm{~b}$ demonstrates. The first driving signal is delivered by a controller, which is 
converted by a Digital-Analog-Converter (DAC) and amplified by a booster, before it reaches the micromirror. Then analog sensing signals from the micromirror are processed by an Analog-Frond-End (AFE) and ADC (Analog-Digital-Converter), before they are demodulated, processed and feed to a Phase-Locked-Loop (PLL), which compose the closed-loop control.

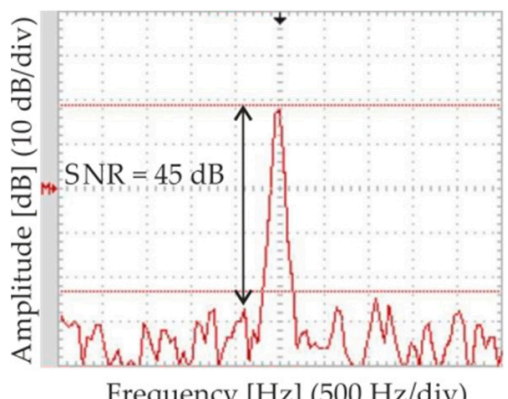

Frequency $[\mathrm{Hz}](500 \mathrm{~Hz} / \mathrm{div})$

(a)

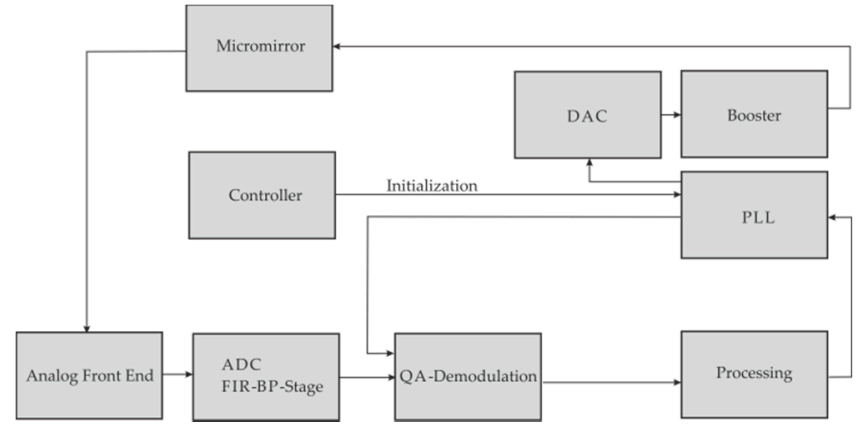

(b)

Figure 5. (a) Measurement results of the PZT sensing signal of design $S$ in frequency domain with a SNR of $45 \mathrm{~dB}$ after 64 averaging process at a mechanical scan angle of $0.7^{\circ}$; (b) Block diagram of the closed-loop control for the piezoelectrically actuated and sensed micromirror.

\section{Outlook and Discussion}

This work presents, large total optical scan angles, high frequencies, high Q-factor have been realized by the presented micromirrors consuming low power. Feedback signals of PZT position sensors of large SNRs were obtained, which enables 12 Bit sensing resolution. However, some design adaptions should be conducted, since at such large scan angles and high frequencies dynamic deformation of the mirror plate is calculably large, which can be simply reduced by using thicker mirror plate (larger thickness of poly-Si) [5]. Moreover, improvements regarding the material long-term stability are also needed to reduce the performance degradation for long driving term.

Conflicts of Interest: The authors declare no conflict of interest.

\section{References}

1. Gu-Stoppel, S.; Kaden, D.; Quenzer, H.J.; Hofmann, U.; Benecke, W. High speed piezoelectric microscanners with large deflection using mechanical leverage amplification. In Proceedings of the 26th European Conference on Solid-State Transducers (EUROSENSOR), Krakow, Poland, 9-12 September 2012; pp. 56-59.

2. Gu-Stoppel, S.; Janes, J.; Quenzer, H.J.; Hofmann, U.; Kaden, D.; Wagner, B.; Benecke, W. Design, fabrication and characterization of low-voltage piezoelectric two-axis gimbal-less microscanners. In Proceedings of the 2013 Transducers Eurosensors XXVII: The 17th International Conference on Solid-State Sensors, Actuators and Microsystems (TRANSDUCERS EUROSENSORS XXVII), Barcelona, Spain,16-20 June 2013; pp. 2489-2492.

3. Rombach, S.; Marx, M.; Gu-Stoppel, S.; Manoli, Y. Low power and highly precise closed-loop driving circuits for piezoelectric micromirrors with embedded capacitive position sensors. In Proceedings of the SPIE 9760 MOEMS Miniaturized Systems XV, 97600Q, San Francisco, CA, USA, 13 February 2016; Volume 9760.

4. Zhang, C.; Zhang, G.; You, Z. A two-dimensional micro scanner integrated with a piezoelectric actuator and piezoresistors. Sensors 2009, 9, 631-644.

5. Gu-Stoppel, S. Entwicklung, Herstellung und Charakterisierung Piezoelektrischer Mikrospiegel; BoD-Books on Demand: Hamburg, Germany, 2016. 